\title{
Tuberculosis transmission in a hospitalised neonate: Need for optimised tuberculosis screening of pregnant and postpartum women
}

\author{
J Zenhäusern, ${ }^{1} \mathrm{MB}$ ChB; A Bekker, ${ }^{2} \mathrm{MB} \mathrm{ChB}, \mathrm{MMed}$ (Paed), Cert Neonatology (SA), PhD; M A Wates, ${ }^{1} \mathrm{MB}$ ChB, FCPaed; \\ H S Schaaf, ${ }^{2}$ MB ChB, MMed, PhD; A Dramowski, ${ }^{3}$ MB ChB, FCPaed, MMed (Paed), Cert Paed ID, PhD \\ ${ }^{1}$ Department of Paediatrics, Karl Bremer Hospital, Cape Town, South Africa \\ ${ }^{2}$ Department of Paediatrics and Child Health, Faculty of Medicine and Health Sciences, Stellenbosch University, and Tygerberg Hospital, \\ Cape Town, South Africa \\ ${ }^{3}$ Academic Unit for Infection Prevention and Control, Division of Health Systems and Public Health, Department of Global Health, \\ Faculty of Medicine and Health Sciences, Stellenbosch University, Cape Town, South Africa
}

Corresponding author: J Zenhäusern (janinezen@gmail.com)

\begin{abstract}
A recent fatal case of confirmed nosocomial tuberculosis (TB) transmission to a neonate in a kangaroo mother care (KMC) unit highlighted the infection risk to hospitalised neonates in South Africa, a high-burden TB setting. The index case was a 9-week-old infant who presented to another hospital's intensive care unit with severe respiratory distress shortly after discharge from the KMC unit. Contact tracing identified that the infant had been exposed to a postpartum woman with undiagnosed pulmonary TB while in the KMC unit. Molecular testing confirmed nosocomial transmission between the index case and the presumed source case in the KMC unit. We describe the subsequent process of tracing other TB-exposed infants and mothers, the difficulty in confirming TB infection/disease in pregnancy, and the provision of isoniazid preventive therapy in this cohort. We discuss the practical implementation of TB screening approaches in maternity and neonatal wards in high-burden TB settings.
\end{abstract}

S Afr Med J 2019;109(5):310-313. DOI:10.7196/SAMJ.2019.v109i5.13789

South Africa (SA) is one of 22 high-burden tuberculosis (TB) countries and has among the highest TB incidence and HIV prevalence rates globally. ${ }^{[1]} \mathrm{SA}$ women in their child-bearing years are at high risk of dual TB and HIV infection, which may pose a significant threat to their newborns if one or both diseases remain undiagnosed and/or untreated. Studies have demonstrated that active screening for TB in antenatal and postnatal care settings is beneficial to both mothers and babies. ${ }^{[2]}$ However, several local studies have demonstrated missed or delayed diagnosis of TB in pregnant SA women, with devastating consequences for them and their babies. ${ }^{[3,4]}$

Despite antenatal TB screening being recommended by the 2014 National Tuberculosis Management Guidelines, ${ }^{[5]}$ routine implementation of screening in busy antenatal clinics is uncommon. Furthermore, no official guidelines exist for TB screening of mothers in kangaroo mother care (KMC) units during the postpartum period, a period that has been shown to carry an increased risk of TB disease. ${ }^{[6]}$ Cases of TB exposure in hospitalised newborns and infants have been documented, particularly from KMC units in TB-endemic settings ${ }^{[7-10]}$ The risk of TB transmission in KMC units is of particular concern, as mothers and infants spend prolonged periods of time in small, often poorly ventilated rooms. ${ }^{[7]} \mathrm{TB}$-exposed infants are at high risk of developing $\mathrm{TB}$ in the absence of appropriate preventive therapy, with up to $50 \%$ of infants developing $\mathrm{TB}, 30 \%$ of whom will have progressive pulmonary or disseminated disease, following exposure and infection. ${ }^{[11]}$

Undiagnosed TB in mothers poses a serious risk of transmission, not only to their own infants but also to other infants in neonatal and paediatric wards, their parents and healthcare workers. ${ }^{[12]}$ There is a clear need to improve TB control strategies in TB-endemic regions, especially in the antenatal, postnatal, neonatal and paediatric healthcare settings. ${ }^{[13]}$ In this case report we report nosocomial $\mathrm{TB}$ transmission to a neonate in a KMC unit, describe the outbreak investigation that ensued, and present recommendations for the prevention of TB transmission in maternity, neonatal and paediatric care settings in SA.

\section{Case report}

The index case

A 9-week-old male infant, born at 34 weeks' gestational age with a birth weight of $2040 \mathrm{~g}$, presented to Tygerberg Hospital with a short history of fast breathing, poor feeding and lethargy. He was one of a set of HIV-unexposed twins who had recently been discharged from Karl Bremer Hospital, a district hospital, following a 17-day stay in the KMC unit. At the time of re-hospitalisation the index case's twin was asymptomatic. The index infant was admitted for investigation and treatment of presumed bacterial sepsis and pneumonia. Intravenous antibiotic therapy (ampicillin and cefotaxime) was commenced. He was transferred to the paediatric intensive care unit for mechanical ventilation after respiratory deterioration. A chest radiograph showed diffuse bilateral pulmonary infiltrates (Fig. 1, A) on the first day of admission. The infant subsequently deteriorated further, requiring high-frequency oscillatory ventilation and nitric oxide therapy. A subsequent chest radiograph 5 days later still showed diffuse bilateral infiltration with a right upper lobe collapse (Fig. 1, B). Poor response to treatment prompted TB investigations; the diagnosis was confirmed on Xpert MTB/RIF testing of a tracheal aspirate (confirming rifampicin-susceptible Mycobacterium tuberculosis). Four-drug antiTB therapy was commenced with isoniazid, rifampicin, ethambutol 


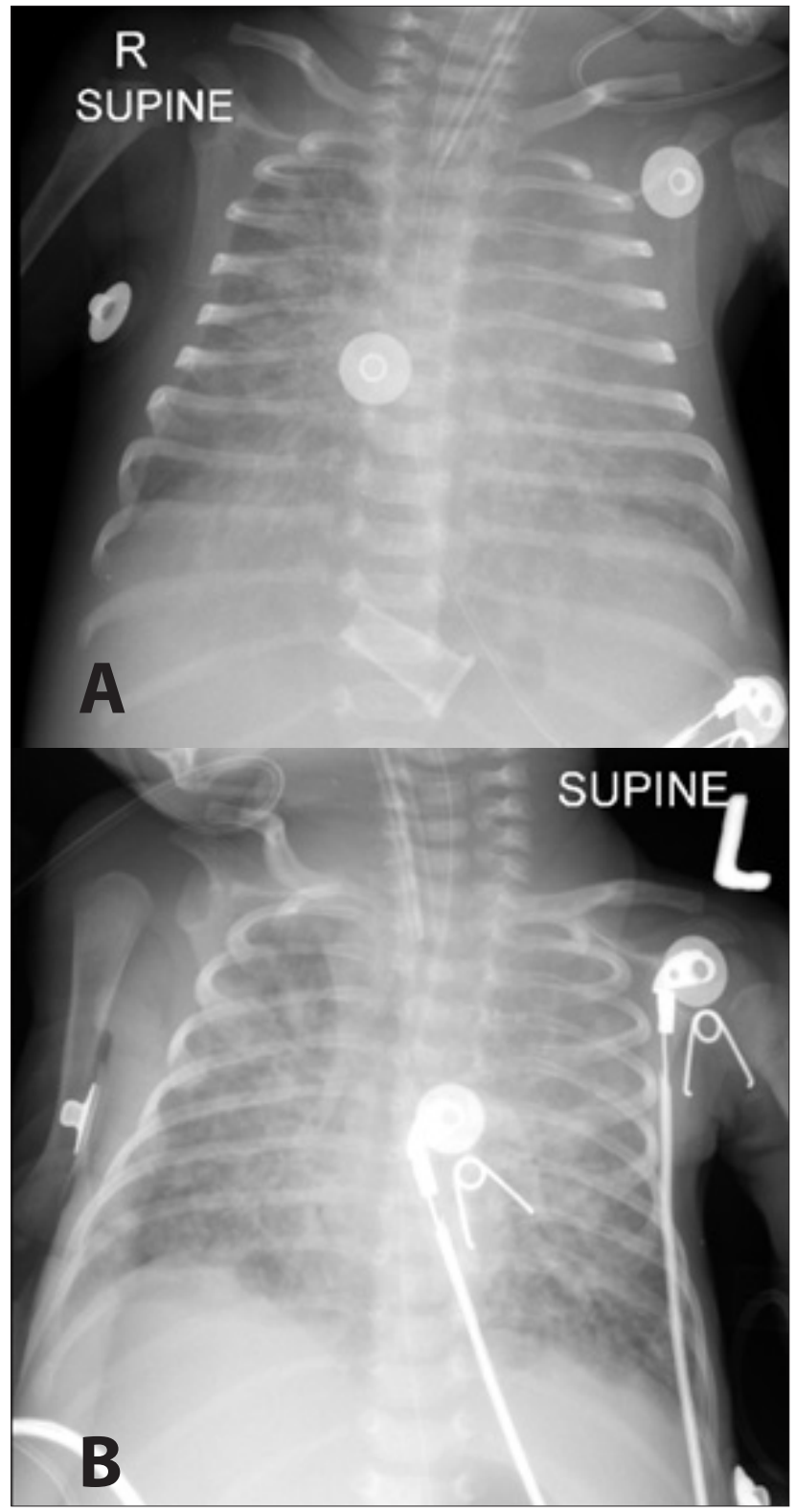

Fig. 1. Chest radiograph of the index patient on admission to the paediatric intensive care unit $(A)$ and 5 days later (B).

and pyrazinamide on day 5 of admission. Owing to further clinical deterioration and increasing ventilation requirements, ganciclovir, high-dose co-trimoxazole, meropenem and vancomycin were also initiated. However, the baby died of multiorgan failure 2 days after the diagnosis of TB. On questioning the family, there were no known household TB source cases. However, the baby's mother reported having shared a room in the KMC unit with another mother who had coughed constantly during her 2-week stay.

\section{The source case}

After obtaining a more detailed history from the mother of the deceased twin and reviewing the hospital records, the possible adult TB source case was identified as a 38-year-old HIV-negative postpartum woman. She had delivered a baby girl (31 weeks' gestational age, $1140 \mathrm{~g}$ ) at Tygerberg Hospital and was transferred to a step-down KMC unit at Karl Bremer Hospital 2 weeks later. At the KMC unit, the source case had slept in the bed adjacent to the index case, his twin and their mother for the full hospital stay. The source case's continuous, productive coughing had been noticed by other KMC unit mothers and allegedly reported to the hospital staff, but unfortunately no further action to investigate her symptoms had been taken. As part of the contact tracing investigation, the source case's medical and laboratory investigations were reviewed and it was discovered that she had recently had sputum samples for TB investigation submitted from a community TB clinic. Her two specimens were microscopy $3+$ smear-positive for acid-fast bacilli and Xpert MTB/RIF-positive for rifampicin-susceptible M. tuberculosis. On contacting the community TB clinic, we learnt that the source case had been recalled to commence TB therapy. Tragically, her own premature infant had been declared dead on arrival at a local clinic the week before, after a short history of cough and respiratory distress (probably TB disease).

\section{Investigation and contact tracing}

Liquid TB culture samples from the index case and the presumed source case were retrieved from the National Health Laboratory Service at Tygerberg Hospital and Green Point TB laboratories, respectively, for comparison. Spoligotyping (a rapid, polymerase chain reaction-based method for genotyping strains of the M. tuberculosis complex that could be used to compare strains) was performed at the Stellenbosch University biomolecular laboratory and confirmed that the strains were identical, belonging to Spoligo International Type 119 (X1-family), confirming nosocomial transmission in the KMC unit. To minimise further potential morbidity and mortality among patients, a contact tracing investigation of mothers, their infants and staff was commenced at the two hospitals where the source case and her premature infant had been hospitalised. Potential TB contacts were defined as infants, mothers and staff whose admission/ service period on the KMC unit coincided with that of the source case (regardless of whether they had shared a room with the source case). The risk of TB transmission was assessed as low at the referral hospital, as the source case had not been an inpatient there and all the infants in the ward to which her baby had been admitted were in incubators, but telephonic follow-up was conducted (30/46 infants were thriving and asymptomatic, 15/46 could not be traced and 1 infant had died from other causes).

Thirty-four mother-infant pairs were identified from the district hospital KMC unit patient register (in total 36 infants, as there were two sets of twins). Contact tracing was conducted using postal communication, telephone calls and home visits by the local police service. Of the 36 infants, 23 (64\%) were recalled and 13 could not be traced despite repeated attempts. A standardised screening method was followed for the mothers (TB symptom screening plus a chest radiograph) and for TB-exposed babies (growth chart weight plotting to identify failure to thrive, symptom screening for cough, lethargy, poor feeding and a chest radiograph). Any radiographic or clinical findings in keeping with possible TB prompted collection of two gastric aspirates for Xpert testing and mycobacterial culture. Of the 23 infants screened, 10 (43\%) required TB investigation with gastric aspirates; all 10 were acid-fast bacilli smear- and mycobacterial culture-negative. After excluding TB disease, 21/23 (91\%) of the traced infants were commenced on a 6-month course of isoniazid preventive therapy (IPT). Only the index case and his sibling $(2 / 23,9 \%)$ had been/were commenced on treatment for TB disease. The twin of the index case was placed on a three-drug TB regimen for 6 months owing to symptoms that included cough, a suspicious chest radiograph and failure to thrive. The source case's baby had died prior to TB investigations, bringing the total number of TB-exposed infants who had confirmed/presumed TB disease to $3 / 36(8 \%)$. 


\section{Discussion}

This case report from an SA neonatal KMC unit demonstrates the vulnerability of neonates to nosocomial TB infection and highlights the need for prompt identification of $\mathrm{TB}$ in pregnant and postpartum women in TB-endemic countries. Despite several published reports of $\mathrm{TB}$ transmission to hospitalised neonates, most TB-endemic settings have yet to implement standardised TB screening that is integrated into existing antenatal, intrapartum, postpartum, neonatal and paediatric healthcare services. ${ }^{[7-10,14,15}$ $\mathrm{TB}$ is also the leading cause of non-obstetricrelated maternal death. ${ }^{[16-18]}$

There are various reasons for the lack of maternal TB screening recommendations and implementation in low-resource, TBendemic countries.

Most countries do not screen routinely for $\mathrm{TB}$ in pregnancy, or report the pregnancy status of female TB patients. In 2011, there were an estimated 216500 cases of TB in pregnant women, with the highest burden in Africa ( $41 \%$ of cases globally). ${ }^{[17]}$ Pregnant women are at increased risk of developing TB disease, ${ }^{[19]}$ but may present nonspecifically and/or have overlap of TB symptoms (fatigue and malaise) with normal pregnancy-related complaints, making the clinical diagnosis of TB in pregnancy difficult. ${ }^{[16,17]}$ As a result, pulmonary $\mathrm{TB}$ and extrapulmonary $\mathrm{TB}$ disease is often not appropriately investigated ${ }^{[16,17,20]}$ with a delay in TB diagnosis of 4 - 26 weeks on average. ${ }^{[16,21-23]}$ Increased awareness of TB risk and clinical presentation in pregnant and postpartum women is needed for both healthcare staff and patients, especially in countries with a high TB burden.

Additional challenges to the prompt diagnosis of TB in pregnant women include poor access to high-quality maternity services, lack of integrated TB screening in maternity services, presentation for antenatal care late in pregnancy, and TB-related stigma. ${ }^{[24-26]}$ Furthermore, the evidence base to inform best practices for maternal TB screening is limited. Most TB programmes use the World Health Organization-recommended TB foursymptom screen to identify the presence of any cough, fever, night sweats or weight loss. ${ }^{[27-29]}$ The presence of any symptom prompts further investigation with $\mathrm{TB}$ sputum smear microscopy/Xpert test and chest radiography when indicated. However, the sensitivity of clinical screening for TB in pregnant women can be as low as $28 \%$, particularly among HIV-infected women. ${ }^{[30]}$ Chest radiography in addition to clinical symptom screening may be a more effective way to identify TB in pregnancy, particularly in settings with a high TB/HIV burden. ${ }^{[12,15,28,29,31]}$

In many cases, discovery of an episode of inadvertent TB exposure in a hospital setting occurs only after recognition of the disease in an index case (often occurring long after the TB exposure event). There are several challenges in deciding on the approach for the exposure/outbreak investigations, including establishing the identity, location and TB treatment status of the source case/s; the infectivity, exposure time and TB drug resistance pattern of the source case/s; the number of exposed infants, staff and parents; the best method to trace and recall affected patients; the best method of screening for TB disease; and whether to provide IPT. For infants with TB disease and no known household TB source case, nosocomial TB exposure during hospitalisation episodes is a strong possibility. A major challenge in our management of this TB-exposure incident was the tracing of babies from the two KMC units, as phone numbers and addresses were incorrect in many hospital folders, and many patients had relocated to other provinces following the birth of their baby Among babies who were successfully traced, underwent TB screening and received IPT, there were no further readmissions and the prophylaxis was well tolerated. Both hospitals' KMC units sensitised staff to screen for TB in postpartum women being admitted on the neonatal wards, and a TB screening protocol was implemented at the district hospital (Fig. 2).

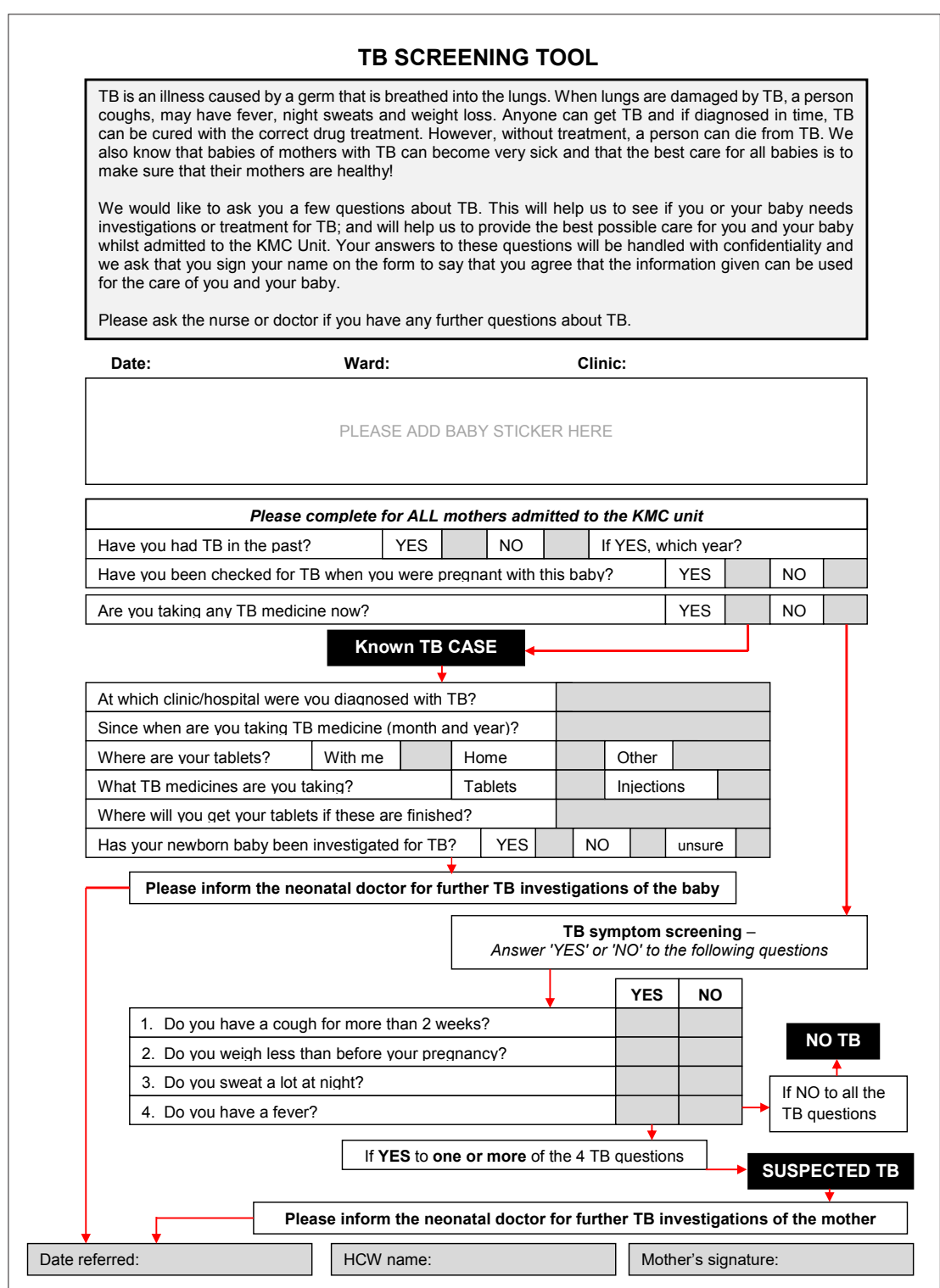

Fig. 2. Example of a maternal tuberculosis screening tool for neonatal wards. 
Table 1. Recommendations to optimise identification of TB in pregnant and postpartum women in countries with a high TB burden

- Increase awareness of TB in pregnancy and in postpartum women among patients and healthcare workers

- Integrate TB screening in routine antenatal, intrapartum, postpartum and early infant care

- In settings where mothers are admitted with their infants, e.g. neonatal/paediatric wards, implement a TB screening algorithm (prior to admission)

- In infants with TB disease and no known household TB contacts, consider the possibility of nosocomial TB transmission during neonatal hospitalisation

$\mathrm{TB}=$ tuberculosis

Inadvertent $\mathrm{TB}$ exposure in neonatal/KMC wards in SA is likely to occur frequently given our high TB incidence and HIV prevalence rates among pregnant women. In order to reduce the risk of TB transmission to hospitalised infants, SA should encourage routine TB screening in antenatal care settings and implement postpartum $\mathrm{TB}$ screening in neonatal wards before women are moved to KMC units (Table 1)

\section{Conclusions}

Hospitalised infants and healthcare staff in TB-endemic settings are at high risk of $\mathrm{TB}$ exposure in healthcare facilities where maternal $\mathrm{TB}$ screening is not routinely conducted. Active TB screening and casefinding programmes for pregnant women are an important way to improve maternal/infant health and survival in TB-endemic settings. Standardised recommendations for TB/HIV screening of pregnant and postpartum women should be developed to ensure prompt identification of infectious individuals and prevent TB transmission in SA healthcare facilities.

\section{Declaration. None.}

Acknowledgements. The authors thank the parents of the index case, who consented to publication of this case report, the clinicians who cared for the index baby in the paediatric intensive care unit (Dr Noor Parker and Prof. Pierre Goussard), the National Health Laboratory Service for obtaining the specimens (Dr Adre Lourens and Cornelia Rautenbach), the Stellenbosch University TB laboratory for performing the spoligotyping (Dr Lizma Streicher and Prof. Rob Warren), the staff of the neonatal units (Sr Fila Abrahams) and infection prevention services at Karl Bremer and Tygerberg hospitals (Sr Anri van Rooyen and Sr Marina Aucamp) who assisted with the contact tracing investigation, and Sr Rula at the local TB clinic. A special thanks also to Dr Liezl Smit, who designed the first version of the TB screening tool.

Author contributions. JZ, $\mathrm{AD}$ and $\mathrm{AB}$ drafted the first version of the manuscript. JZ and MW conducted the contact investigation at the district hospital KMC unit. AB, HSS and AD supervised the contact tracing at the referral hospital. All authors gave input on and approved the final version of the manuscript.

Funding. None.

Conflicts of interest. None.

1. World Health Organization. Global Tuberculosis Report 2017. http://www.who.int/tb/publications global_report/en/ (accessed 20 June 2018).

2. Ndwiga C, Birungi $\mathrm{H}$, Undie $\mathrm{CC}$, Weyenga $\mathrm{H}$, Sitienei J. Feasibility and effect of integrating tuberculosi screening and detection in postnatal care services: An operations research study. BMC Health Serv Res 2013;13:99. https://doi.org/10.1186/1472-6963-13-99.6

3. Bekker A, du Preez K, Schaaf HS, Cotton MF, Hesseling AC. High tuberculosis exposure among neonates in a high tuberculosis and human immunodeficiency virus burden setting. Int J Tuberc Lun Dis 2012;16(8):1040-1046. https://doi.org/10.5588/ijtld.11.0821

4. Adhikari M, Pillay T, Pillay DG. Tuberculosis in the newborn: An emerging disease. Pediatr Infect Dis J 1997;16(12):1108-1112
5. National Department of Health, South Africa. National Tuberculosis Management Guidelines 2014. http://www.tbonline.info/media/uploads/documents/ntcp_adult_tb-guidelines-27.5.2014.pdf (accessed 23 August 2018)

6. Zenner D, Kruijshaar ME, Andrews N, Abubakar I. Risk of tuberculosis in pregnancy: A national, Zenner D, Kruijshaar ME, Andrews N, Abubakar I. Risk of tuberculosis in pregnancy: A national,
primary care-based cohort and self-controlled case series study. Am J Respir Crit Care Med 2012:185(7):779-784. https://doi.org/10.1164/rccm.201106-10830C

7. Heyns L, Gie RP, Goussard P, Beyers N, Warren RM, Marais BJ. Nosocomial transmission of Mycobacterium tuberculosis in kangaroo mother care units: A risk in tuberculosis-endemic areas. Acta Paediatr 2006;95(5):535-539. https://doi.org/10.1080/08035250600636560

8. Grisaru-Soen G, Savyon M, Sadot E. Congenital tuberculosis and management of exposure in neonatal and pediatric intensive care units. Int J Tuberc Lung Dis 2014;18(9):1062-1065. https://doi. org $/ 10.5588 /$ ijtld. 14.0160

9. Winters A, Agerton TB, Driver CR, Trieu L, O'Flaherty T, Munsiff SS. Congenital tuberculosis and management of exposure in three neonatal intensive care units. Int J Tuberc Lung Dis 2010;14(12):1641-1643.

10. Ahn JG, Kim DS, Kim KH. Nosocomial exposure to active pulmonary tuberculosis in a neonatal intensive care unit. Am J Infect Control 2015;43(12):1292-1295. https://doi.org/10.1016/j, ajic.2015.07.020

11. Marais BJ, Gie RP, Schaaf HS, et al. The clinical epidemiology of childhood pulmonary tuberculosis: A critical review of literature from the pre-chemotherapy era. Int J Tuberc Lung Dis 2004:8(3):278-285.

12. Schaaf HS, Donald PR Scott F Maternal chest radiography as supporting evidence for the dignosis of

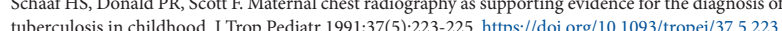

tuberculosis in childhood. J Trop Pediatr 1991;37(5):223-225. https://doi.org/10.1093/tropej/37.5.223 Hesseling AC, Cotton MF, Jennings T, et al. High incidence of tuberculosis among HIV-infected infants: Evidence from a South African population-based study highlights the need for improved

14. Schaaf HS, Collins A, Bekker A, Davies PD. Tuberculosis at extremes of age. Respirology 4. Schaaf HS, Collins A, Bekker A, Davies PD. Tuberculosis at
2010;15(5):747-763. https://doi. org $/ 10.1111 /$ 1 1440-1843.2010.01784. X

15. Schechner V, Lessing JB, Grisaru-Soen G, et al. Preventing tuberculosis transmission at a maternity . Schechner V, Lessing JB, Grisaru-Soen $\mathrm{G}$, et al. Preventing tuberculosis transmission at a maternity
hospital by targeted screening radiography of migrants. J Hosp Infect 2015;90(3):253-259. https://doi. org/10.1016/j.jhin.2015.03.008

16. Jain KP, Chaudhari PB. Need for increased clinical awareness of tuberculosis during pregnancy and puerperium in India. Int J Reprod Contracept Obstet Gynecol 2016;5(12):4106-4111. https://doi. org/10.18203/2320-1770.ijrcog20164312

17. Sugarman J, Colvin C, Moran AC, Oxlade O. Tuberculosis in pregnancy: An estimate of the global burden of disease. Lancet Glob Health 2014;2(12):e710-e716. https://doi.org/10.1016/S2214109X(14)70330-4

18. Habib ZG, Dayyab FM, Sanda A, et al. Role of GeneXpert MTB/Rif assay in diagnosing tuberculosis in pregnancy and puerperium. Case Rep Infect Dis 2015; article ID 794109. https://doi. org $/ 10.1155 / 2015 / 794109$

19. Bates M, Ahmed Y, Kapata N, Maeurer M, Mwaba P, Zumla A. Perspectives on tuberculosis in pregnancy. Int J Infect Dis 2015;32:124-127. https://doi.org/10.1016/j.ijid.2014.12.014

20. Mathad JS, Gupta A. Tuberculosis in pregnant and postpartum women: Epidemiology, management and research gaps. Clin Infect Dis 2012;55(11):1532-1549. https://doi.org/10.1093/cid/cis732

21. Gupta A, Nayak U, Ram M, et al.; Byramjee Jeejeebhoy Medical College-Johns Hopkins University Study Group. Postpartum tuberculosis incidence and mortality among HIV-infected women and their Study Group. Postpartum tuberculosis incidence and mortality among HIV-infected women and their

22. Llewelyn M, Cropley I, Wilkinson RJ, Davidson RN. Tuberculosis diagnosed during pregnancy: A prospective study from London. Thorax 2000;55(2):129-132. https://doi.org/10.1136/thorax.55.2.129

A prospective study from London. Thorax 2000;55(2):129-132. https://doi.org/10.1136/thorax.55.2.129
23. Kothari A, Mahadevan N, Girling J. Tuberculosis and pregnancy - results of a study in a high prevalence area in London. Eur J Obstet Gynecol Reprod Biol 2006;126(1):48-55. https://doi. org/10.1016/j.ejogrb.2005.07.025

24. Kali PB, Gray GE, Violari A, Chaisson RE, McIntyre JA, Martinson NA. Combining PMTCT with active case finding for tuberculosis. J Acquir Immune Defic Syndr 2006;42(3):379-381. https://doi. org/10.1097/01.qai.0000218434.20404.9c

25. Turnbull ER, Kancheya NG, Harris JB, Topp SM, Henostroza G, Reid SE. A model of tuberculosis screening for pregnant women in resource-limited settings using Xpert MTB/RIF. J Pregnancy 2012; article ID 565049. https://doi.org/10.1155/2012/565049

26. Murray EJ, Bond VA, Marais BJ, Godfrey-Faussett P, Ayles HM, Beyers N. High levels of vulnerability and anticipated stigma reduce the impetus for tuberculosis diagnosis in Cape Town, South Africa. Health Policy Plan 2013;28(4):410-418. https://doi.org/10.1093/heapol/czs072

27. World Health Organization. Systematic Screening for Active Tuberculosis - Principles and Recommendations. Geneva: WHO, 2013. http://www.who.int/tb/publications/Final_TB_Screening_ guidelines.pdf (accessed 20 July 2018).

28. Kosgei RJ, Ndavi PM, Ongech JO, et al. Symptom screen: Diagnostic usefulness in detecting pulmonary tuberculosis in HIV-infected pregnant women in Kenya. Public Health Action 2011;1(2):30-33. https://
tong tuberculosis in HIV-infected pres

29. Kosgei RJ, Szkwarko D, Callens S, et al. Screening for tuberculosis in pregnancy: Do we need more than Kosgei RJ, Szkwarko D, Callens S, et al. Screening for tuberculosis in pregnancy: Do we need more than
a symptom screen? Experience from western Kenya. Public Health Action 2013;3(4):294-298. https://

30. Hoffmann CJ, Variava E, Rakgokong M, et al. High prevalence of pulmonary tuberculosis but low sensitivity of symptom screening among HIV-infected pregnant women in South Africa. PLoS One 2013;8(4):e62211. https://doi.org/10.1371/journal.pone.006221

31. Good JT Jr, Iseman MD, Davidson PT, Lakshminarayan S, Sahn SA. Tuberculosis in association with pregnancy. Am J Obstet Gynecol 1981;140(5):492-498. 